\title{
Remote sensing and modeling of lightning caused long recovery events within the lower ionosphere using VLF/LF radio wave propagation
}

\author{
E. D. Schmitter \\ University of Applied Sciences Osnabrueck, 49076 Osnabrueck, Germany \\ Correspondence to: E. D. Schmitter (e.d.schmitter@hs-osnabrueck.de)
}

Received: 7 November 2013 - Revised: 25 March 2014 - Accepted: 29 March 2014 - Published: 11 November 2014

\begin{abstract}
On the 4 November 2012 at 3:04:27 UT a strong lightning in the midst of the North Sea affected the propagation conditions of VLF/LF transmitter radio signals from NRK (Iceland, $37.5 \mathrm{kHz}$ ) and GBZ (UK, $19.58 \mathrm{kHz}$ ) received at $5246^{\circ} \mathrm{N} 8^{\circ} \mathrm{E}$ (NW Germany). The amplitude and phase dips show a recovery time of 6-12 min pointing to a LOng Recovery Early VLF (LORE) event. Clear assignment of the causative return stroke in space and time was possible with data from the WWLLN (Worldwide Lightning Location Network). Based on a return stroke current model the electric field is calculated and an excess electron density distribution which decays over time in the lower ionosphere is derived. Ionization, attachment and recombination processes are modeled in detail. Entering the electron density distribution in VLF/LF radio wave propagation calculations using the LWPC (Long Wavelength Propagation Capability) code allows to model the VLF/LF amplitude and phase behavior by adjusting the return stroke current moment. The results endorse and quantify the conception of lower ionosphere EMP heating by strong - but not necessarily extremely strong - return strokes of both polarities.
\end{abstract}

\section{Introduction}

In the early morning hours of the 4 November 2012 a heavy thunderstorm was active in the North Sea area. Figure 1 shows the location of a strong return stroke (marked as S2 in the center of the yellow circles with $90 \mathrm{~km}$ and $240 \mathrm{~km}$ radius) recorded by the WWLLN (Worldwide Lightning Location Network) at 3:04:27 UT. According to Rodger et al. (2006) WWLLN is especially suited to study the occurrence and impacts of high peak-current lightning. Figure 2 displays the amplitude and phase dips of the GBZ signal together with 4 WWLLN time ticks fitting in the time range 2.5-5:00 UT and locations near the center of the circles shown in the map together with energy estimates. Two return strokes had very large energies ( 340 and $885 \mathrm{~kJ}$ as reported by WWLLN). The return stroke marked as S2 at 3:04:27 UT changed the night time ionospheric propagation conditions for about $12 \mathrm{~min}$. Our further considerations refer to this event.

The WWLLN data of this return stroke are:

- date and time in UT: 2012/11/4, 03:04:27.018495

- lat, lon in fractional degrees (geographic coordinates): $54.8586,003.2279$

- residual fit error in microseconds (always <30): 20.0

- number of WWLLN stations participating in the location fit: 17

- RMS energy (in Joules) of the stroke (from $1.3 \mathrm{~ms}$ waveform sampling between 7 and $18 \mathrm{kHz}$ ): 340069.91

- energy uncertainty (energy error of the fit in Joules): 166388.33

- subset of stations between 1000 and $8000 \mathrm{~km}$ from the stroke whose energy data were used in the energy estimate: 9 . 


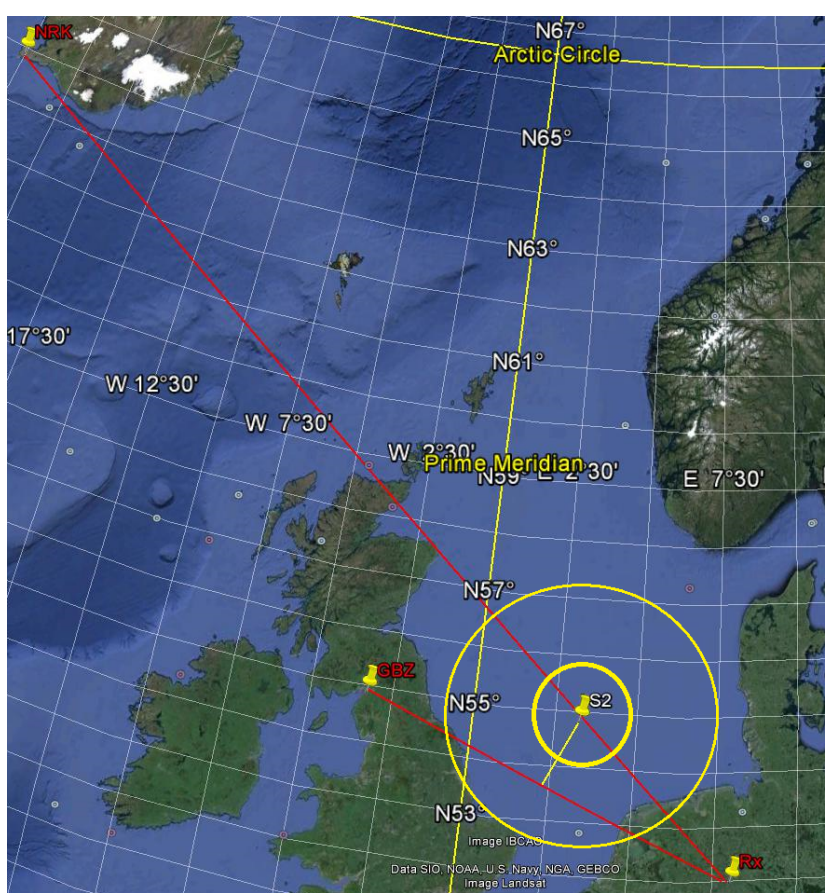

Figure 1. The Position S2 at the center of the yellow circles refers to a return stroke with an energy of $340 \mathrm{~kJ}$ according to WWLLN at 20121104, 3:04:27 UT. It is also marked as S2 in Fig. 2. The inner circle has a radius of $90 \mathrm{~km}$, where our EMP model predicts a maximum excess ionization in the night time lower ionosphere of the 2.35 fold of the undisturbed nighttime background. The outer circle has a radius of $240 \mathrm{~km}$, where the excess ionization is the 1.7 fold of the night time background. Also shown are the great circle VFL propagation paths NRK (Iceland) $-52^{\circ} \mathrm{N} 8^{\circ} \mathrm{E}$ and GBZ (UK) $-52^{\circ} \mathrm{N} 8^{\circ} \mathrm{E}$ (marked as Rx) crossing the lightning stroke affected area. The shortest distances of the paths to the $\mathrm{S} 2$ stroke foot point are $8 \mathrm{~km}$ for NRK and $143 \mathrm{~km}$ for GBZ.

In the case presented in this paper amplitude and phase react within $3 \mathrm{~s}$, which is the monitoring time period, see Fig. 3, probably much faster. This points to a ionospheric disturbance caused by the reported lightning return stroke S2. With regard to a recovery time of about $12 \mathrm{~min}$ the event is "slow", implicating persistent ionization. Cotts and Inan (2007) distinguish 3 types of long recovery events: long-amplitude/short-phase recovery (Type 1), longamplitude/long-phase recovery (Type 2), and step-change events (Type 3 ) in which the amplitude does not return to pre-event levels. With a recovery time of $12 \mathrm{~min}$ in amplitude and phase, an amplitude dip of $-5 \mathrm{~dB}$ and a phase change of $35^{\circ}$ our reported event is of type 2 with regard to the GBZ signal, Figs. 2, 3, and 11. Because of ionospheric fluctuations the recovery time is determined with an error bound of 2-3 min. With regard to NRK the event is clearly detectable but too weak to classify it, especially the phase dip drowns in the background noise, Fig. 12. We show in this paper, that the large difference of the effects on both paths is modeled correctly by the assumption of an EMP action.
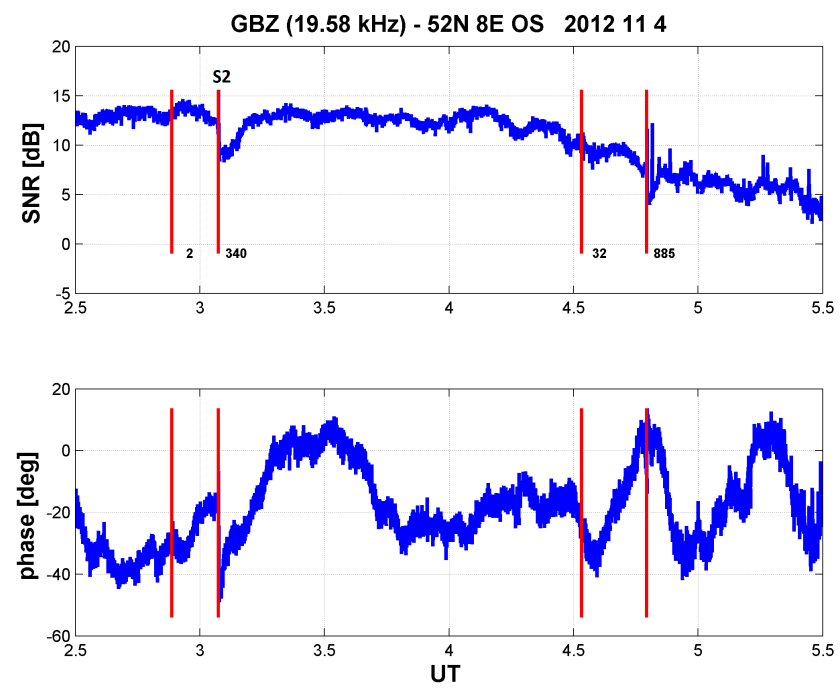

Figure 2. 20121104: monitored amplitude (signal to noise ratio, SNR) and phase of the GBZ signal $(19.58 \mathrm{kHz})$ at $52^{\circ} \mathrm{N} 8^{\circ}$ E. Ticks and numbers: return stroke times and energies $(\mathrm{kJ})$ provided by WWLLN. The position of S2 is marked in Fig. 1.

With regard to the persistent ionization responsible for the VLF events in question by changing propagation conditions along the signal paths mainly 2 causes are discussed:

1. Generation of positive and negative ions at low altitudes $(<60 \mathrm{~km})$ by gigantic blue jets. Lehtinen and Inan (2007) use a five constituent model of the stratospheric/lower ionospheric chemistry indicating that ionization produced in gigantic blue jets may persist for tens of minutes, especially at stratospheric altitudes, the primary reason for it being the slow process of the mutual neutralization of stratospheric ions.

2. Ionization within the upper D-layer of the ionosphere by the electric field pulse of a return stroke as part of its radiated EMP (Electro Magnetic Pulse) at nighttime. Ionization persistence in this case is a consequence of the low atmospheric density slowing down attachment and recombination. The ionized volume at heights of about $90 \mathrm{~km}$ can also produce an optically visible elve ("Emissions of Light and VLF perturbations due to EMP Sources", Fukunishi et al., 1996). Early VLF perturbations associated with elves have been detected successfully on several occasions, Inan et al. (1996, 2010); Mika et al. (2006). It is proposed, that LORES are caused by large and long-lived electron density enhancements in the upper D-region ionosphere caused by intense positive or negative cloud to ground $(\mathrm{CG}+/-)$ lightning discharges, Haldoupis et al. (2012, 2013).

In the case presented here, a related sferic is clearly identified by the WWLLN. In case solely a gigantic blue jet would be the cause of the VLF event without an accompanying return stroke, the question arises, whether a blue jet can produce a sferic like signature as detected by the WWLLN. There is no endorsing case study known to us. The second 
possibility would be a return stroke directly related to a blue jet in time and space, where the stroke radiates the sferic and the blue jet generates the ionization. With regard to both possibilities the EMP action of a single return stroke is the simpler assumption and we show in this paper that it can explain our data.

We take the opportunity given by our recorded event to show that a return stroke of positive or negative polarity with sufficient but not extremely high peak current moment and fast current rise is able to generate a LORE and to explain our data with regard to the disturbed amplitude and phase.

In the following we discuss the generation and the decay of an excess electron density distribution caused by a return stroke E field and the resulting implications for the VLF/LF radio wave propagation conditions. We start with an analytical expression for the free electric field generated by the current of a single return stroke of constant vertical channel length as a source. Entering the lower ionosphere the electric field is attenuated but can enhance electron density by impact ionization if it is strong enough. The product of vertical return stroke channel length and return stroke current can be adjusted to model our VLF/LF radio wave propagation data.

A similar approach is discussed in Lay et al. (2010). They use a 2-D FDTD (Finite Difference Time Domain) numerical model to calculate the propagating electric field from a current function. Marshall et al. (2008) discuss a 3-D FDTD model of the multi stroke EMP-ionosphere interaction and a 2-D FDFD model of the VLF signal propagation through a disturbed region.

Within an analytical model, where all physical relations are presented, the effect of parameters on the results can be discussed mathematically in a more comprehensible way than relying on numerical methods like FDTD/FD. So we think, that our continuously analytical model is more transparent for the discussion of the effect of important parameters controlling the generation and the decay of the excess electron density. With regard to the VLF signal propagation we rely on the full wave solutions of the LWPC (Long Wave Propagation Capability) code, Ferguson (1998), that has been proved to be reliable with many applications.

In this paper we firstly explain our remote sensing method and continue with describing the lightning current source, the electric field propagation and its attenuation. After that the calculation of the electron density profiles with regard to their generation and decay is explained. Then the VLF/LF radio wave propagation calculations using the modeled electron density profiles are described. We finish with some results and conclusions.

\section{Remote sensing}

Remote sensing of lower ionosphere conditions (bottomside sounding) by monitoring low and very low frequency radio signal propagation is a well known method for several
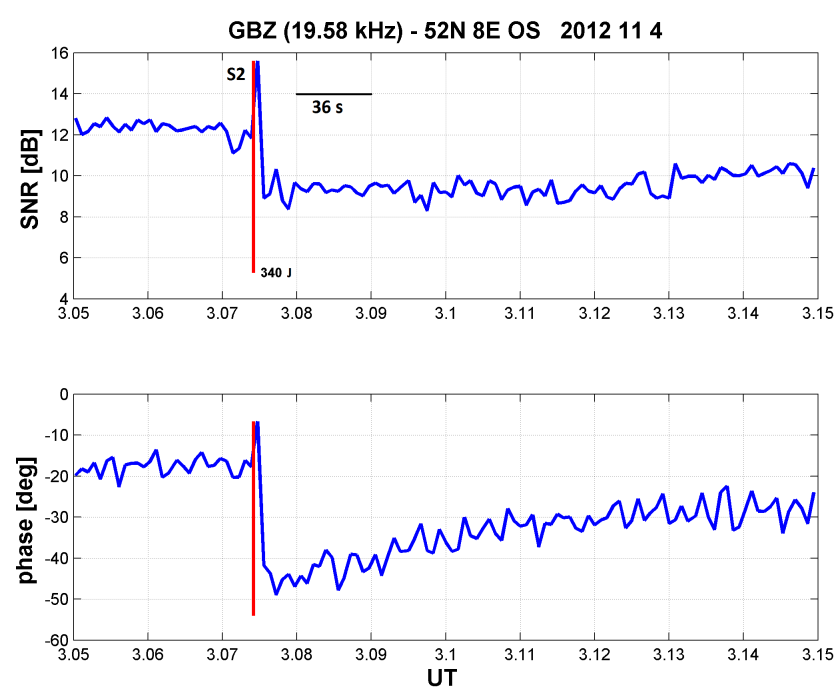

Figure 3. 20121104: monitored amplitude (signal to noise ratio, SNR) and phase of the GBZ signal $(19.58 \mathrm{kHz})$ at $52^{\circ} \mathrm{N} 8^{\circ} \mathrm{E}$; zoomed detail from Fig. 2 around return stroke event S2. Amplitude and phase react within less than $3 \mathrm{~s}$ (= data acquisition period).

decades. MSK (Minimum Shift Keying) transmitters prove as useful in this respect because of their constant amplitude emissions. We have analyzed the signal amplitude and phase variations of 2 transmitters, NRK/TFK $\left(37.5 \mathrm{kHz}, 63.9^{\circ} \mathrm{N}\right.$ $22.5^{\circ} \mathrm{W}$, Iceland $)$ and GBZ $\left(19.58 \mathrm{kHz}, 54.9^{\circ} \mathrm{N} 3.3^{\circ} \mathrm{W}\right.$, UK) received at a mid latitude site $\left(52^{\circ} \mathrm{N} 8^{\circ} \mathrm{E}\right)$ with great circle distances of 2210 and $800 \mathrm{~km}$, respectively. The receivers (one for each transmitter) have been set up by us with ferrite coils oriented for the maximum signal amplitude of the horizontal magnetic field. After pre-amplification a stereo sound card computer interface with $192 \mathrm{kbit}$ sample rate is used. The second channel is fed with the 1-s pulse of a GPS receiver. Our software reads each second a $170 \mathrm{~ms}$ signal train and extracts within the narrow MSK bandwidth $(200 \mathrm{~Hz}$ in our cases) amplitude and phase with regard to the rising GPS-pulse flank yielding a time synchronization better than $100 \mathrm{~ns}$, corresponding to phase detection errors of $0.7^{\circ}$ $(19.58 \mathrm{kHz})$ and $1.4^{\circ}(37.5 \mathrm{kHz})$. For the amplitude the signal to noise ratio (SNR) is recorded. $\mathrm{SNR}=0 \mathrm{~dB}$ is defined by the averaged signal level received during transmitter maintenance drop outs. The time stability of both transmitters proves to be sufficient for continuous day and night monitoring not only of the amplitude but also of the phase. Our phase detection algorithm for MSK signals records phases between -90 and $+90^{\circ}$.

The NRK path starts within the auroral domain and most of its way proceeds through the sub-auroral domain. The GBZ path proceeds at the equator ward boundary of the subauroral domain. These propagation paths to our mid latitude site prove well suited to study lower ionosphere forcing from above with regard to auroral and sub-auroral particle precipitation (Schmitter, 2010) as well as forcing from below 

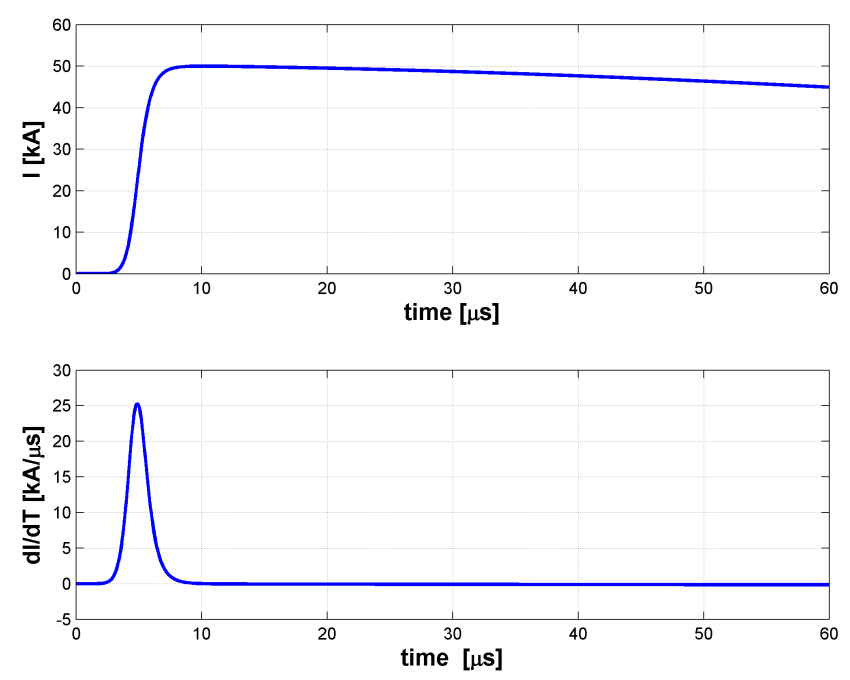

Figure 4. The "early" part of the model current of the return stroke and its derivative. The main contribution to the generated electromagnetic far field results from the current time derivative (bottom panel).

by planetary wave activity (Schmitter, 2012), which is more prominent with higher latitudes. They also yield relevant information about solar flares (Schmitter, 2013) and in the case described here about the effects of strong lightning return strokes in the lower ionosphere in the North Sea area.

\section{The lightning $\mathbf{E}$ field}

We model the return stroke current according to a Heidler type function (Heidler et al., 1999)

$I(t)=I_{0} \frac{\left(\frac{t}{\tau_{1}}\right)^{n}}{1+\left(\frac{t}{\tau_{1}}\right)^{n}}\left(e^{-\left(\frac{t}{\tau_{2}}\right)^{2}}+\eta e^{-\left(\frac{t}{\tau_{2}}\right)^{2}}\right)$

$I_{0}$ is defined to yield a given maximum current $I_{\max }$, in our example $50 \mathrm{kA}$.

The factor $\frac{\left(\frac{t}{\tau_{1}}\right)^{n}}{1+\left(\frac{t}{\tau_{1}}\right)^{n}}$ with time constant $\tau_{1}=5 \mu$ s and $n=$ 10 lets the current rise within $6 \mu$ s from zero to peak with a maximum rate of $25 \mathrm{kA} / \mu \mathrm{s}$. The second factor $\left(e^{-\left(\frac{t}{\tau_{2}}\right)^{2}}+\right.$ $\left.\eta e^{-\left(\frac{t}{\tau_{2}}\right)^{2}}\right)$ with time constant $\tau_{2}=150 \mu \mathrm{s}$ and $\eta=0.6$ models a much slower decay with a peak to half-peak time of $40 \mu \mathrm{s}$ and a peak to peak/4 time of $150 \mu$ s.

As the sign of the current - and the generated electric field accordingly - does not enter our electron density calculations, see Sect. 4, we keep it positive throughout. The detailed form of Eq. (1) is important only with regard to $(d I / d t)_{\max }$ and $I_{\max }$ because the pulse like current derivative together with the maximum current reached and the channel length are the main drivers for the EMP reaching the lower ionosphere, cp. Eq. (2). See Fig. 4 showing the relevant (early)

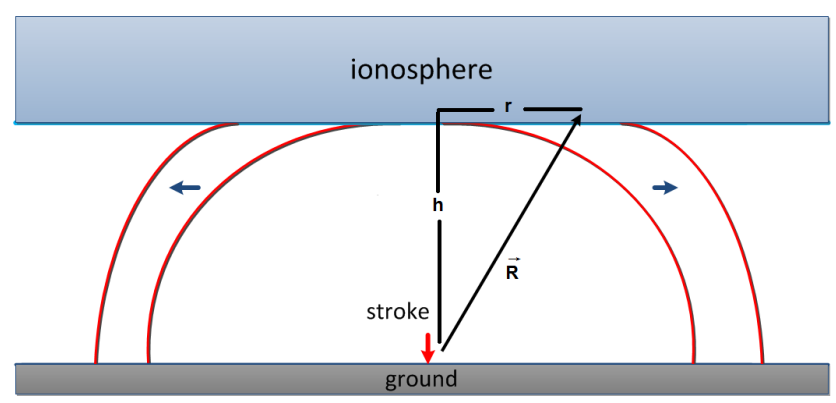

Figure 5. Return stroke EMP (electric field part) affecting VLF propagation conditions by ionization; geometry of the situation.

part of the current and its derivative according to this model function. The charge transfer (integrated current) and energy transfer $\left(R \int I(\tau)^{2} d \tau\right.$, i.e. average total channel resistance times the action integral) is of minor importance for the EMP generation. The Heidler type functions model especially the rising part better than other functions, see Rakov and Uman (1998) for a discussion.

The free electric field above a perfectly conducting ground (well satisfied with sea water) in cylinder coordinates $\left(r, h, R:=\sqrt{r^{2}+h^{2}}\right)$, cp. Fig. 5, with vertical channel length $L$ is given by Uman (2001):

$\boldsymbol{E}(r, h)=\left(\begin{array}{c}E_{r} \\ E_{h}\end{array}\right)=$

$\frac{L}{2 \pi \varepsilon_{0}}\left[\left(\frac{\int I d t}{R^{5}}+\frac{I}{c R^{4}}\right)\left(\begin{array}{c}3 r h \\ 2 h^{2}-r^{2}\end{array}\right)+\frac{d I / d t}{c^{2} R^{3}}\left(\begin{array}{c}r h \\ -r^{2}\end{array}\right)\right]$

Obviously for the far field radiation the important parameter is $L \frac{d I}{d t}$.

Within the lower ionosphere the E field is attenuated with height $h$ according to:

$\boldsymbol{E}(h)=\boldsymbol{E}\left(h_{0}\right) e^{-\int_{h_{0}}^{h} \frac{1}{s(z)} d z}$

$s(h)=\frac{2}{\sigma(h)} \sqrt{\frac{\epsilon_{0}}{\mu_{0}}}$

$s(h)$ is the skin depth in a poor conductor at height $h$. "Poor" means $\sigma<\epsilon_{0} \omega$, $\omega$ being the typical maximum radian frequency of the process in question which is of the order of $2 \pi \cdot 10 \mathrm{kHz}$ for a return stroke. Significant conductivity contributions can be expected for $h>h_{0}=60 \mathrm{~km}$.

In the VLF/LF range conductivity is related to electron density as $\sigma=\epsilon_{0} \frac{\omega_{p}^{2}}{f_{c}}=\frac{e^{2} n_{e}}{m_{e} f_{c}},\left(\omega_{p}\right.$ : plasma frequency, $f_{c}$ : collision frequency, $e$ : elementary charge, $m_{e}$ electron mass).

So, E field attenuation within a stratified ionosphere with electron density profile $n_{e}(h)$ and collision frequency profile $f_{c}(h)$ can be expressed as

$\boldsymbol{E}(h)=\boldsymbol{E}\left(h_{0}\right) e^{-\gamma \int_{h_{0}}^{h} \frac{n_{e}(z)}{f_{c}(z)} d z}$ 
with $\gamma:=\frac{e^{2}}{2 m_{e}} \sqrt{\frac{\mu_{0}}{\epsilon_{0}}}$.

We show in this paper that the proposed model together with a radiating channel length of $L=5 \mathrm{~km}$ - describing a strong, but not exceptionally strong return stroke - can explain our data. See Gamerota et al. (2012); Uman (2001) for a discussion of the statistical distribution of measured lightning current characteristics.

\section{Modeling electron density profiles}

Undisturbed conductivity is parametrized according to

$\sigma(h)=\sigma_{0} e^{\beta\left(h-h^{\prime}\right)}$

using the Wait \& Spies (Wait and Spies, 1964) 2-parameter model for the lower ionosphere (about 60-95 km height) with regard to $h^{\prime}$ (effective height, $\mathrm{km}$ ) and $\beta$ (profile steepness, $1 / \mathrm{km}), \sigma_{0}=\sigma\left(h=h^{\prime}\right)=2.22 \cdot 10^{-6} \mathrm{~S} / \mathrm{m}$.

By fitting our nighttime data on the date in question using LWPC modeling (described in Sect. 5), we have determined undisturbed nighttime values of $h^{\prime}=84 \mathrm{~km}$ and $\beta=0.67 \mathrm{~km}^{-1}$ which we will use as our background conditions for this modeling study.

From Eq. (6) together with the collision frequency profile $f_{c}(h)=f_{0} e^{-h / H}, f_{0}=1.816 \cdot 10^{11} \mathrm{~Hz}$ and the relation between conductivity and electron density appropriate for VLF/LF sounding $\sigma=\epsilon_{0} \frac{\omega_{p}^{2}}{f_{c}}=\frac{e^{2} n_{e}}{m_{e} f c}\left(\omega_{p}\right.$ : plasma frequency) we get the classic Wait \& Spies (Wait and Spies, 1964) electron density parametrization of the lower ionosphere:

$n_{e}=n_{0} e^{-h / H} e^{\beta\left(h-h^{\prime}\right)}$

with $n_{0}=1.43 \cdot 10^{13} \mathrm{~m}^{-3}$ and scale height $H=1 / 0.15=$ $6.67 \mathrm{~km}$ corresponding to an isothermal atmosphere with $T=230 \mathrm{~K}$.

For nighttime background conditions, we use $h^{\prime}=84 \mathrm{~km}$ and $\beta=0.67 \mathrm{~km}^{-1}$ as determined from the data (Fig. 6).

When the (attenuated) electric field pulse reaches a point within the lower ionosphere, depending on the pulse field strength $\mathrm{E}$ and neutral density, impact ionization can take place, which locally increases electron density.

A fast concurrent electron loss mechanism is dissociative attachment $\left(e+\mathrm{O}_{2} \rightarrow \mathrm{O}^{-}+\mathrm{O}\right)$. Three body attachment processes $\left(e+\mathrm{O}_{2}+A \rightarrow \mathrm{O}_{2}^{-}+A, A=\mathrm{O}_{2}\right.$ and $\left.A=\mathrm{N}_{2}\right)$ have time scales $>1 \mathrm{~s}$ and need not be considered in the few milliseconds of the electron excess generation process, BarringtonLeigh (2000); Pasko et al. (1997). Three body attachment and recombination are however the dominating processes that let the excess electron density decay after the first milliseconds within seconds to minutes.

So, starting with the undisturbed electron density distribution, $n_{e}(r, h, t)$ develops in time according to the rate equation:

$$
\frac{\partial}{\partial t} n_{e}(r, h, t)=\left[v_{i}(E(r, h, t), h)-v_{a}(E(r, h, t), h)\right] n_{e}(r, h, t)
$$
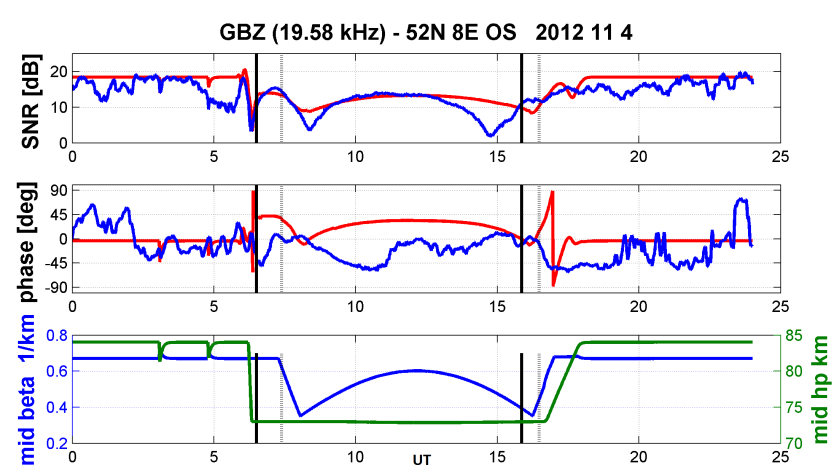

Figure 6. Received (blue) and modeled (red) VLF signal from GBZ, 20121104. Upper 2 panels: recorded amplitude and phase behavior (blue) and result of the propagation calculation (red). Bottom panel: equivalent electron density profile height $h^{\prime}$ and steepness $\beta$ of the lower ionosphere; for our calculations we use the undisturbed night time values: $h^{\prime}=84 \mathrm{~km}$ and $\beta=0.67 \mathrm{~km}^{-1}$. The course of the $h^{\prime}$, $\beta$ profile values given in this panel refers to the middle of the GBZ propagation path. With regard to the propagation calculations using LWPC the electron density height profile is recalculated in $50 \mathrm{~km}$ long segments along the path. The $x$ axis is labeled with UT (hours).

yielding the EMP generated excess electron density distribution:

$n_{\mathrm{EMP}}(r, h)=n_{e}(r, h, \triangle t)-n_{e}(r, h, 0) \quad \triangle t \approx 2 \mathrm{~ms}$

$v_{i}, v_{a}\left(\mathrm{~s}^{-1}\right)$ are the impact ionization and dissociative attachment coefficients respectively in the presence of an electric field $\mathrm{E}$.

The coefficients have the form $v_{i, a}=n_{n} f_{i, a}\left(E / n_{n}\right)$ with electric field strength $E$ and neutrals density $n_{n}$. BarringtonLeigh (2000) and Pasko et al. (1997) give detailed information about the series expansions of $v_{i}, v_{a}$. The functions are fits to experimental data. So, electron gain by impact ionization and loss by dissociative attachment in the rate Eq. (8) are proportional to the product of electron density and neutrals density. The proportionality functions $f_{i, a}$ scale with $E / n_{n}$, i.e. high electric field strength at low heights (high $n_{n}$ ) yields the same values for $f_{i, a}$ as a low field strength at large heights (low $n_{n}$ ). In passing by we note that in gas discharge physics processes often scale with $E / n_{n}$, because mean electron energy as a discharge driver is a function of $E / n_{n}$ which has its own unit: 1 Townsend $=10^{-21} \mathrm{Vm}^{2}$.

During the integration of Eq. (8) with increasing electron density the electric field attenuation also increases because conductivity is directly proportional to electron density. So Eq. (5) has to be re-evaluated self-consistently with each time step. We also note that because $v_{i}, v_{a}$ depend on the absolute value of the E field only, the polarity of the return stroke current does not turn up in this model. Figure 7 gives an example of the numerical solution of Eq. (8) at $r=90 \mathrm{~km}$ and height $h=93 \mathrm{~km}$. The electric field of the return stroke reaches this 

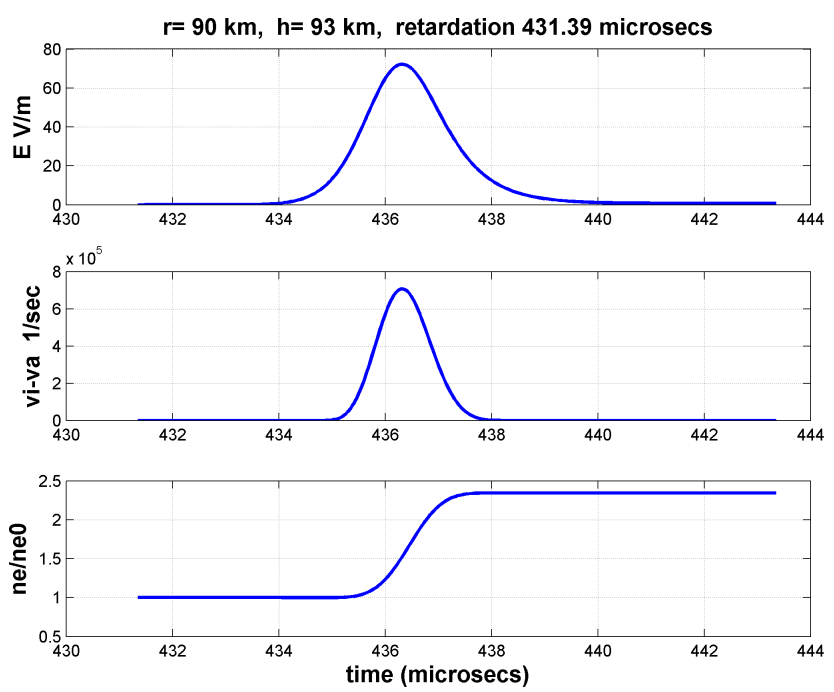

Figure 7. Time development of the electric field (top panel), the difference between impact ionization (gain) coefficient and dissociative attachment (loss) coefficient (middle panel) and relative electron density excess according to the integration of Eq. (8) at $r=90 \mathrm{~km}$ and $h=93 \mathrm{~km}$, bottom panel. EM radiation from the stroke foot point ( $r=0, h=0$ ) needs $431 \mu$ s to reach this point.

point after $431 \mu$ s. The top panel shows the electric field with a maximum of $75 \mathrm{~V} / \mathrm{m}$, the middle panel the difference of electron gain and loss coefficients $v_{i}-v_{a}\left(s^{-1}\right)$ and the bottom panel the electron density increase relative to the background. The main activity takes place within about 3-4 $\mu$ s.

After $2 \mathrm{~ms}$ the excess electron density is fully developed across a diameter of more than $500 \mathrm{~km}$ and Fig. 8 displays the electron density distribution relative to the undisturbed background, $\frac{n_{e}(r, h, t=2 \mathrm{~ms})}{n_{e}(r, h, t=0)}$. To get a more detailed impression Fig. 10 presents the height profiles of the electron density enhancement with regard to the undisturbed background at several radial distances. At any radial distance the maximum is reached at $h=93 \mathrm{~km}$.

The electron density distribution $n_{e}(r, h, t)\left(\mathrm{m}^{-3}\right)$ now decays according to the rate equation, Lay et al. (2010):

$$
\frac{\partial}{\partial t} n_{e}(r, h, t)=q_{\mathrm{EMP}}(r, h, t)+q_{u}(h)-\beta_{i}(h) n_{e}-\alpha(h) n_{e}^{2}
$$

$q_{u}(h)$ : ion-pair production $\left(\mathrm{m}^{-3} \mathrm{~s}^{-1}\right)$, not related to EMP, during nighttime mainly caused by geocorona scattered Lyman Alpha radiation and cosmic rays. We assume the steady state condition $q_{u}(h)=\beta_{i}(h) n_{e 0}(h)+\alpha(h) n_{e 0}^{2}(h)$ with the undisturbed night time electron density $n_{e 0}$ from Eq. (7) with $h^{\prime}=84 \mathrm{~km}$ and $\beta=0.67 \mathrm{~km}^{-1}$.

$\beta_{i}(h)$ : night time three body attachment coefficient $\left(\mathrm{s}^{-1}\right)$, taken from Rodger et al. (1998))

$\alpha(h)$ : night time recombination coefficient $\left(\mathrm{m}^{3} \mathrm{~s}^{-1}\right)$ with regard to $\mathrm{O}_{2}^{+}$and hydrated ions, taken from Rodger et al. (2007)

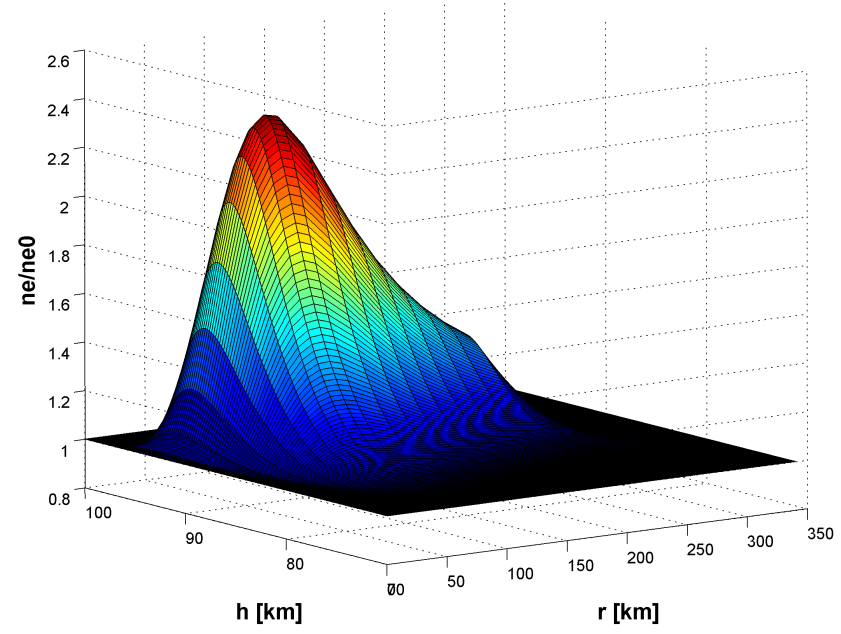

Figure 8. The modeled relative electron density excess distribution around the return stroke position $(r=0, h=0)$ as a result from the EMP. $n_{e 0}$ is the night time undisturbed Wait and Spies electron density profile with $h^{\prime}=84 \mathrm{~km}$ and $\beta=0.67 \mathrm{~km}^{-1}$. See Fig. 9 with regard to profile slices at different radial distances from the stroke position.

$q_{\mathrm{EMP}}$ : ion pair production $\left(\mathrm{m}^{-3} \mathrm{~s}^{-1}\right)$ caused by the lightning return stroke EMP:

$q_{\mathrm{EMP}} \approx n_{\mathrm{EMP}}(r, h) \frac{1}{\triangle t}$

for the first time step $\Delta t$ of Eq. (10), $q_{\mathrm{EMP}}=0$ later.

All numerical integrations are done using the classic Runge-Kutta algorithm. With Eq. (8) a time step of 10 nanoseconds is used, with Eq. (10) a time step of $10 \mathrm{~s}$.

\section{Propagation calculations}

For frequencies below the plasma frequency $\sqrt{\frac{e^{2} n_{e}}{\epsilon_{0} m_{e}}}$ the space between the conducting earth ground and the ionosphere behaves like a leaky waveguide. For the VLF/LF range the diffuse upper waveguide boundary is formed by the lower ionosphere $(60-90 \mathrm{~km}$ height). Propagation calculation in our case is the task to calculate at a ground based receiver position the signal field amplitude and phase of a ground based transmitter (vertical electric and horizontal magnetic field components). Besides transmitter radiated power and antenna characteristics the essential input to the calculation are the conductivities of the waveguide along the great circle propagation path. We used the LWPC (Long Wave Propagation Capability) code, Ferguson (1998), for this purpose. It includes a world wide map of the ground conductivities. For the ionospheric conductivity $\left(\sigma=\frac{e^{2} n_{e}}{m_{e} f_{c}}\right)$ the collision frequency and electron density height profiles along the path have to be provided. The collision frequency profile is usually assumed to be constant along the path: $f_{c}(h)=$ 


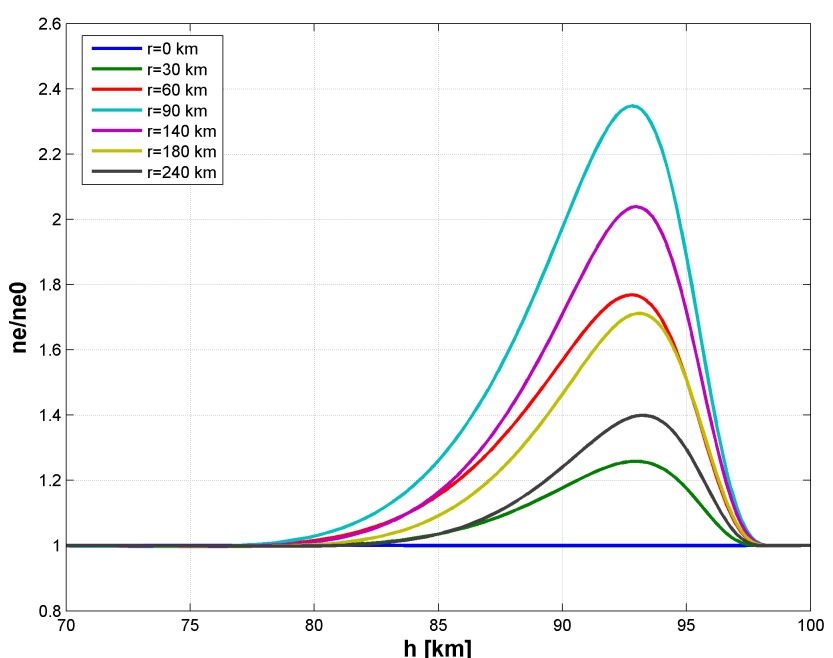

Figure 9. Height profiles of the modeled electron density excess distribution caused by the return stroke EMP at radial distances of $0,30,60,90,140,180$ and $240 \mathrm{~km}$ from the return stroke foot point (relative to the night time undisturbed profile, cp. Fig. 8 for a 3-Dplot).The maximum value of the 2.35 fold of the undisturbed background value is reached at a distance of $90 \mathrm{~km}$. The local maxima at any distance from the stroke foot point are reached at an altitude of $93 \mathrm{~km}$.

$f_{0} e^{-h / H}, f_{0}=1.816 \cdot 10^{11} \mathrm{~Hz}, H=6.67 \mathrm{~km}$. It may be modulated by gravity or planetary waves (Schmitter, 2012). The electron density $n_{e}$ changes along the path with sun zenith angle, activity of the sun (e.g. solar flares, Schmitter, 2013) and because of local forcing from above (particle precipitation) or below, e.g. lightning EMP. For the undisturbed $n_{e^{-}}$ profile the Wait and Spies function with parameters $h^{\prime}$ and $\beta$, Eq. (7), is a useful approximation. With the night time profile $\left(h^{\prime}=84 \mathrm{~km}\right.$ and $\left.\beta=0.67 \mathrm{~km}^{-1}\right)$ as initial value (for each height $h$ ) Eq. (10) integrates the EMP disturbed profile $n_{e}(r, h, t)$ along the path for the times after the stroke. The ionization $q_{\mathrm{EMP}}$ results from Eq. (11), where $n_{\mathrm{EMP}}$ in turn evaluates from Eqs. (8), (9). We have performed calculations in one minute intervals. The propagation path is divided in $50 \mathrm{~km}$ segments. Each segment is provided with the proper electron density height profile for the time step in question and the radial distance of the segment center to the stroke foot point.

LWPC then does a full wave calculation resulting in the vertical electric field amplitude and phase at the receiver site, the horizontal magnetic field data being directly proportional. To allow comparisons with our recorded data amplitude results are displayed in $\mathrm{dB}$ above the noise level, cp. Figs. 6, 11. Phases are folded into the range $-90 . .+90^{\circ}$ as only this range is detected by our algorithm.

In order to compare results with other work, the disturbed electron density profile can be fitted with a Wait and Spies profile with effective reflection height $h^{\prime}$ and steepness $\beta$, see Fig. 10 for an example. For the VLF/LF propagation only the

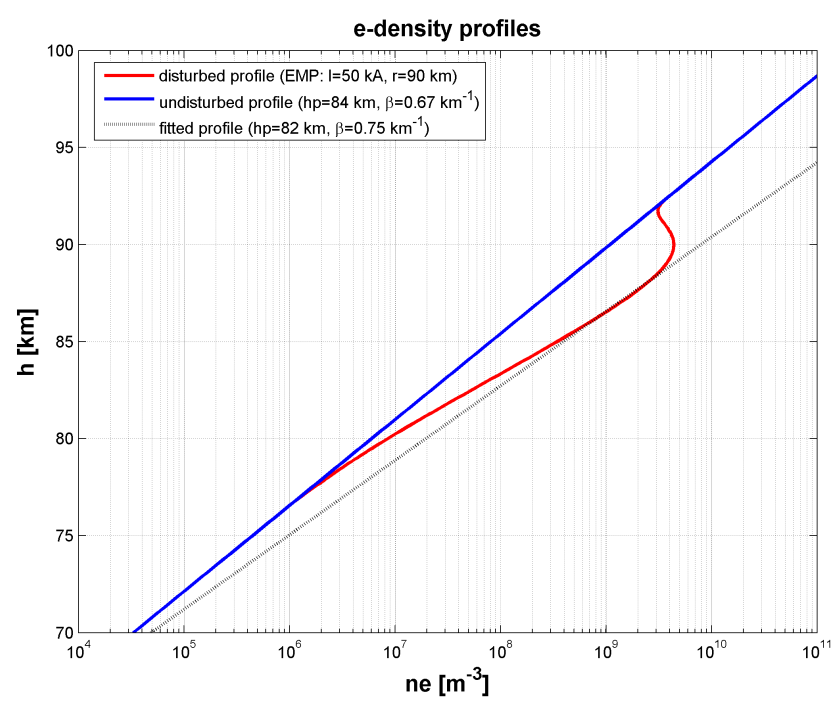

Figure 10. The undisturbed Wait and Spies night time density profile (blue) together with the EMP disturbed electron density (red) at a distance of $r=90 \mathrm{~km}$ from the return stroke foot point, where the electron excess assumes its maximum, cp. Figs. 8 and 9. The dotted line is a fit of a Wait and Spies profile to the disturbed electron density profile. With regard to the undisturbed night time profile values, $h^{\prime}$ is decreased and $\beta$ is increased, cp. Fig. 11, bottom panel.

lower part of the profile is of importance (heights $h<87 \mathrm{~km}$ in the nighttime example). It is therefore comprehensible that LWPC calculations using the fitted $\left(h^{\prime}, \beta\right)$ profiles (with a new fit at any time step and path segment distance from the stroke) yield the same results with regard to amplitude and phase compared to those using the integrated profiles according to Eq. (10). In Figs. 6 and 11 we have made use of this concept. Results are typically sensitive to an $h^{\prime}$-change of $>0.2 \mathrm{~km}$ and a $\beta$ (steepness) change of $>0.01 \mathrm{~km}^{-1}$.

\section{Modeling results}

The maximum current moment $L I_{\max }$, i.e. vertical channel length times maximum current is adjusted to $5 \mathrm{~km} *$ $50 \mathrm{kA}=2.5 \cdot 10^{8} \mathrm{Am}$ to fit the observed signal amplitude and phase dips, Fig. 11. This value corresponds to a strong, but not exceptionally strong return stroke. This goes into the same direction as NaitAmor et al. (2013) which observed that low peak current strokes can cause long recovery events. We note that the maximum current moment $L I_{\max }$ is the only free parameter used in our model: Eq. (2) contains $L I(t)$ and $I(t)$ is linearly proportional to $I_{\max }$ (in Eq. $1, I_{0}$ is adjusted to yield a given value of $I_{\max }$ ). A variation of $L I_{\max }$ of up to $\pm 5 \%$ does not distort significantly the fit to the data. We have to keep in mind, that the main driver for the EMP is the current derivative maximum, i.e. the steep rising part of the current model function, Eq. (1). Given the $I(t)$-model function, 


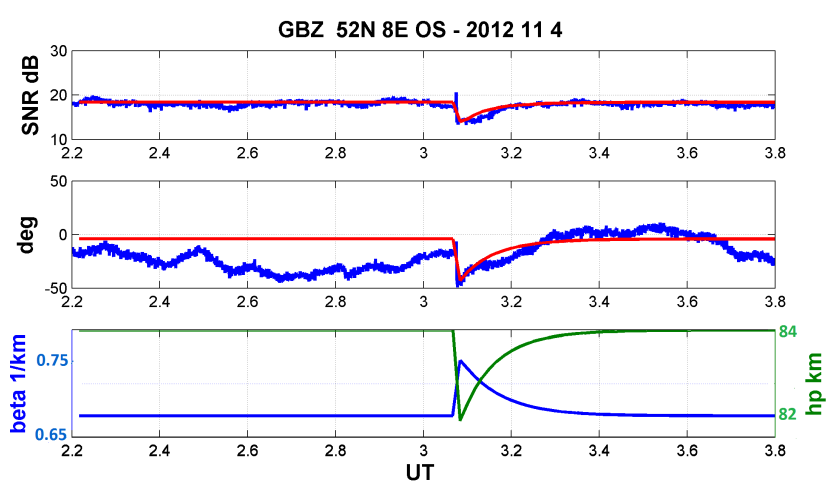

Figure 11. The return stroke S2 at 20121104 3:04:27 UT and its effect with regard to the GBZ path (enlarged detail from Fig. 6). Upper 2 panels: recorded amplitude and phase behavior (blue) and the result of the propagation calculation (red). Bottom panel: equivalent values of the effective height $h^{\prime}$ and steepness $\beta$ of the lower ionosphere electron density profile consistent with the propagation calculation. The electron density profile height decreases sharply by $2 \mathrm{~km}$ and recovers within the next $12 \mathrm{~min}$. The profile steepness increases slightly and recovers within the same time. The course of the profile values given in this panel refers to the middle of the GBZ propagation path. For the propagation calculation leading to the results (red) in the upper 2 panels the electron density profile is recalculated using Eq. (10) within $50 \mathrm{~km}$ long segments along the propagation path taking into account the segment distance to the stroke foot point.

$I_{\max }$ not only defines the current maximum but also uniquely defines the current derivative maximum.

The sign of the current does not occur in the model, so both CG+ and CG- return strokes can be responsible for long recovery events. The maximum disturbed electron density of the 2.35 fold of the background density is reached at $93 \mathrm{~km}$ height and at $90 \mathrm{~km}$ radial distance from the return stroke foot point. Directly above the return stroke is a deep minimum, so that the excess electron density geometrically is a toroidal disc well known from elves, which are the recombination glow revealing the electric field entering the lower ionosphere.

Modeling of the amplitude and phase of the NRK signal $(37.5 \mathrm{kHz}$, Iceland) with the same parameters, i.e. the same excess electron density distribution, also shows very good agreement with the data, see Fig. 12. There is significantly less effect of the EMP with regard to the NRK propagation path, despite this path completely traverses the stroke affected area and at its nearest point is much closer to the return stroke location than the GBZ path ( $8 \mathrm{~km}$ for NRK, compared to $143 \mathrm{~km}$ with GBZ, Fig. 1). At first sight this is an astonishing behavior, but we can argue that the NRK propagation is dominated by the first mode (or one hop mode) because of NRKs rather large distance. The ionization of the lower ionosphere in the middle of the path therefore most strongly affects signal amplitude and phase but the corresponding area is out of reach of the stroke, Fig. 1. With GBZ

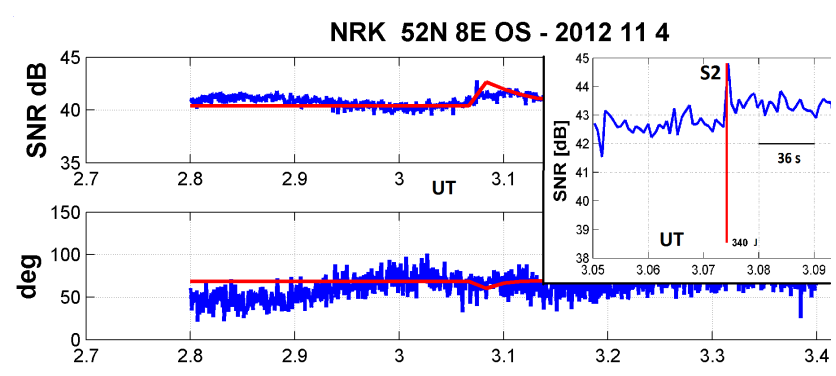

Figure 12. The return stroke S2 at 20121104 3:04:27 UT and its effect with regard to the NRK path. Upper and lower panel: recorded amplitude and phase behavior (blue) and the result of the propagation calculation (red). The inlay shows the zoomed amplitude (monitoring resolution: $3 \mathrm{~s}$ ). The effect of the return stroke with regard to this propagation path is much weaker than with the GBZ path (Fig. 11). The different effects on both propagation paths are correctly described by our calculations within the modeling resolution of one minute.

Fig. 1 shows that nearly the whole path is located within the excess electron density cloud and a strong reaction of the VLF signal is expected. To get detailed predictions about polarity and strengths of the amplitude and phase disturbances full wave propagation calculation is necessary to include all relevant wave modes, as is done using the LWPC. This is especially important for short propagation paths (GBZ). The importance of this point is also stressed in NaitAmor et al. (2013).

In our model we assumed the action of a single return stroke. In principle the decaying electron density cloud may have been formed by a sequence of return strokes within the same flash causing an accumulating effect, see Lay et al. (2010), despite that WWLLN detected only one stroke in our example case.

\section{Conclusions}

Monitoring VLF/LF MSK transmitters also in this investigation proves as an inexpensive way to sense forcing of the lower ionosphere, in this case with regard to lightning return strokes causing long recovery events in VLF/LF signal data. Signal amplitude and phase dips which are synchronous with a strong return stroke are successfully modeled by an EMP from of a single vertical return stroke with current moment $2.5 \cdot 10^{8}$ Am that generated electron densities up to 2.35 times larger than the undisturbed night time value (i.e. up to $4.5 \cdot 10^{9} \mathrm{~m}^{-3}$ ). The toroidal electron density cloud at a center height of $93 \mathrm{~km}$ had a diameter of more than $500 \mathrm{~km}$ affecting VLF/LF propagation conditions depending on the position of the propagation path. The focal point of our work is modeling the physical processes leading to the generation of excess electron density profiles caused by an electric field pulse in the night time lower ionosphere and the following 
decay of the excess electron densities. Our results endorse and quantify the conception of lower ionosphere EMP heating by strong return strokes (Inan et al., 1996; Pasko et al., 1997; Marshall et al., 2008). Whether a return stroke causes a detectable long recovery event firstly depends on the position of the electron density disturbance with regard to the whole propagation path and secondly on the maximum current momentum and current rise time. There is no dependence on the return stroke polarity in our model.

Strong and long lasting events like the one described in this paper are quite rare in the range of the North Sea and North Atlantic. Anyway our example endorses the observation of Salut et al. (2012) that long recovery events are attributed predominantly to lightning discharges occurring over the sea. So with regard to long recovery events it is especially promising to analyze propagation paths crossing regions with frequent and strong thunderstorm activity over the sea, for example the Mediterraneans during winter time, see Haldoupis et al. (2013), or the Indian ocean, as reported by Salut et al. (2012).

Acknowledgements. The author would like to thank R. Holzworth, University of Washington, Seattle, for providing the WWLLN data (http://wwlln.net/).

Edited by: M. Förster

Reviewed by: G. Stober and two anonymous referees

\section{References}

Barrington-Leigh, C. P.: Fast Photometric Imaging of High Altitude Optical Flashes above Thunderstorms, chap. 2.4.2, Ionization, Dissertation, Stanford University, Sep. 2000.

Cotts, B. R. T. and Inan, U. S.: VLF observation of long ionospheric recovery events, Geophys. Res. Lett., 34, L14809, doi:10.1029/2007GL030094, 2007.

Cummer, S. A., Inan, U. S., and Bell, T. F.: Ionospheric D region remote sensing using VLF radio atmospherics, Radio Sci., 33, 1781-1792, doi:10.1029/98RS02381, 1998.

Ferguson, J. A.: Computer Programs for Assessments of LongWavelength Radio Communications, Version 2.0, Technical Document May 1998, SPAWAR Systems Center, San Diego, USA, 1998.

Fukunishi, H., Takahashi, Y., Kubota, M., Sakanoi, K., Inan, U. S., and Lyons, W. A.: Elves: Lightning-induced transient luminous events in the lower ionosphere, Geophys. Res. Lett., 23, 21572160, 1996.

Gamerota, W. R., Elisme, J. O., Uman, M. A., and Rakov, V. A.: Current Waveforms for Lightning Simulation, IEEE T. Electromagn. C., 54, 880-888, doi:10.1109/TEMC.2011.2176131, Aug. 2012.

Haldoupis, C., Cohen, M., Cotts, B., Arnone, E., and Inan, U. S.: Long-lasting D-region ionospheric modifications, caused by intense lightning in association with elve and sprite pairs, Geophys. Res. Lett., 39, L16801, doi:10.1029/2012GL052765, 2012.
Haldoupis, C., Cohen, M., Arnone, E., Cotts, B., and Dietrich, S.: The VLF fingerprint of elves: Step-like and long-recovery early VLF perturbations caused by powerful CG lightning EM pulses, J. Geophys. Res. Space Phys., 118, 5392-5402, doi:10.1002/jgra.50489, 2013.

Heidler, F. J., Cvetic, M., and Stanic, B. V.: Calculation of lightning current parameters, IEEE Trans. Power Del., 4, 399-404, 1999.

Inan, U. S., Sampson, W. A., and Taranenko, Y. N.: Space-time structure of optical flashes and ionization changes produced by lightning-EMP, Geophys. Res. Lett., 23, 133-136, 1996.

Inan, U. S., Cummer, S. A., and Marshall, R. A.: A survey of ELF and VLF research on lightning-ionosphere interactions and causative discharges, J. Geophys. Res., 115, A00E36, doi:10.1029/2009JA014775, 2010.

Lay, E. H., Rodger, C. J., Holzworth, R. H., Cho, M., and Thomas, J. N.: Temporal-spatial modeling of electron density enhancement due to successive lightning strokes, J. Geophys. Res., 115, A00E59, doi:10.1029/2009JA014756, 2010.

Lehtinen, N. G. and Inan, U. S.: Possible persistent ionization caused by giant blue jets, Geophys. Res. Lett., 34, L08804, doi:10.1029/2006GL029051, 2007.

Marshall, R. A., Inan, U. S., and Chevalier, T. W.: Early VLF perturbations caused by lightning EMP-driven dissociative attachment, Geophys. Res. Lett., 35, L21807, doi:10.1029/2008GL035358, 2008.

Mika, A., Haldoupis, C., Neubert, T., Su, H. T., Hsu, R. R., Steiner, R. R., and Marshall, R. A.: Early VLF perturbations observed in association with elves, Ann. Geophys., 24, 2179-2189, 2006, http://www.ann-geophys.net/24/2179/2006/.

NaitAmor, S., Cohen, M. B., Cotts, B. R. T., Ghalil, H., AlAbdoadaim, M. A., and Graf, K.: Characteristics of long recovery early VLF events observed by the North African AWESOME Network, J. Geophys. Res. Space Phys., 118, 5215-5222, doi:10.1002/jgra.50448, 2013.

Pasko, V. P., Inan, U. S., Bell, T. F., and Taranenko, Y. N.: Sprites produced by quasi-electrostatic heating and ionization in the lower ionosphere, J. Geophys. Res., 102, 4529-4561, 1997.

Rakov, V. A. and Uman, M. A.: Review and Evaluation of Lightning Return Stroke Models Including Some Aspects of Their Application, IEEE T. Electomagn. C., 40, 403-426, 1998.

Rodger, C. J., Molchanov, O. A., and Thomson, N. R.: Relaxation of transient ionization in the lower ionosphere, J. Geophys. Res., 103, 6969-6975, 1998.

Rodger, C. R. and McCormick, R. J.: Remote sensing of the upper atmosphere by VLF, in: Sprites, Elves and Intense Lightning Discharges, NATO Sci. Ser. II Mat., 225, 167-190, 2006.

Rodger, C. J., Werner, S., Brundell, J. B., Lay, E. H., Thomson, N. R., Holzworth, R. H., and Dowden, R. L.: Detection efficiency of the VLF World-Wide Lightning Location Network (WWLLN): initial case study, Ann. Geophys., 24, 3197-3214, doi:10.5194/angeo-24-3197-2006, 2006.

Rodger, C. R., Clilverd, M. A., Thomson, N. R., Gamble, R. J., Seppaelae, A., Turunen, E., Meredith, N. P., Parrot, M., Sauvaud, J. A., and Berthelier, J. J.: Radiation belt electron precipitation into the atmosphere: Recovery from a geomagnetic storm, J. Geophys. Res., 112, A11307, 12 p., doi:10.1029/2007JA012383, 2007.

Salut, M. M., Abdullah, M., Graf, K. L., Cohen, M. B., Cotts, B. R. T., and Kumar, S.: Long recovery VLF perturbations associ- 
ated with lightning discharges, J. Geophys. Res., 117, A08311, doi:10.1029/2012JA017567, 2012.

Schmitter, E. D.: Remote auroral activity detection and modeling using low frequency transmitter signal reception at a mid latitude site, Ann. Geophys., 28, 1807-1811, doi:10.5194/angeo-281807-2010, 2010.

Schmitter, E. D.: Data analysis of low frequency transmitter signals received at a mid latitude site with regard to planetary wave activity, Adv. Radio Sci., 10, 279-284, doi:10.5194/ars-10-279-2012, 2012.

Schmitter, E. D.: Modeling solar flare induced lower ionosphere changes using VLF/LF transmitter amplitude and phase observations at a mid latitude site, Ann. Geophys., 31, 765-773, doi:10.5194/angeo-31-765-2013, 2013.
Strangeways, H. J.: Lightning, trimpis and sprites, in: Review of radio science 1993-1996, edited by: Stone, W. R., 741-780, Oxford University Press, Oxford, 1996.

Uman, M. A.: The Lightning Discharge, Dover Books on Physics, 2001, unabridged republication, originally published: Orlando: Academic Press, ISBN 0-486-41463-9 (pbk.), 1987.

Wait, J. R. and Spies, K. P.: Characteristics of the Earth-ionosphere waveguide for VLF radio waves, National Bureau of Standards, Boulder, Colorado, NBS Tech. Note, 300, Dec. 30th, 1964. 